\title{
The recurrent nevus phenomenon ${ }^{*}$
}

\author{
Rafaella Daboit Castagna ${ }^{1}$ \\ Raíssa Massaia Londero Chemello ${ }^{1}$
}

Juliana Mazzoleni Stramari ${ }^{1}$

DOI: http:/ / dx.doi.org/10.1590/abd1806-4841.20176190

\begin{abstract}
Recurrent melanocytic nevus is a proliferation of melanocytes arising from a melanocytic nevus removed partially. Asymmetry and irregular pigmentation may lead to misdiagnosis of melanoma. We report a patient presented with a lesion on the lower abdomen, which was removed by shave excision. Anatomopathological examination revealed an intradermal melanocytic nevus. Two months later, a new irregular hyperpigmented lesion appeared in the surgical scar. Histopathology of the excisional biopsy revealed a recurrent melanocytic nevus. Recurrent melanocytic nevus manifests as a scar with hyper or hypopigmented areas, linear streaking, stippled pigmented halos, and/or diffuse pigmentation patterns. Histologically, the dermoepidermal junction and the superficial dermis show melanocytic proliferation overlying the scarred area. When a pathological report of the previous lesion is not available, complete excision is the gold standard. Otherwise, regular dermoscopic monitoring is a therapeutic option. The present report emphasizes the importance of histopathological examination of the excised material - even in cases of suspected benign lesions - and warns patients about the possibility of recurrence in case of incompletely removed lesions.
\end{abstract}

Keywords: Ambulatory surgical procedures; Nevus; Histology

\section{INTRODUCTION}

Recurrent melanocytic nevus is defined as the proliferation of remaining melanocytes that occurs after the partial removal of a melanocytic nevus, which leads to repigmentation at the site of a previous excision. Shave excisions - which are usually performed because of excellent cosmetic results - are often related to recurring nevi. Recognizing this phenomenon is very important, especially because recurrent melanocytic nevi display asymmetric borders and irregular pigmentation in the majority of the cases, which may lead to diagnostic confusion with melanoma. ${ }^{1-4}$

Histopathological study of melanocytic lesions represents one of the most difficult and controversial issues in the pathology domain. Although it is the gold standard for the diagnosis of melanocytic lesions, eventually, the histopathological study may not be conclusive. Diagnostic error may also occur. This is a problem of significant relevance, given the magnitude of the controversial diagnosis of melanoma in case of a benign lesion - or the false diagnosis of benign lesion in case of a melanoma. ${ }^{1,2}$

The present case report aims to highlight the importance of histopathological study of excised melanocytic lesions, even in the face of a diagnostic suspicion of a benign lesion.

\section{CASE REPORT}

A 48-year-old female patient presented for evaluation of a hyperpigmented lesion on the abdomen present since childhood. The lesion had become voluminous some months before, displaying multiple brownish tones. Physical examination revealed a sessile and multilobulated papule, ranging in color from normochromic to dark brown, measuring about $1 \mathrm{~cm}$, located on the right lower quadrant of the abdomen. In view of the diagnostic hypothesis of melanocytic nevus, the lesion was removed by shave excision. Histopathological examination revealed an intradermal melanocytic nevus with the presence of nevus cells in the deep surgical margin. The patient returned two months later after noticing a new dark spot at the site of the previous excision. Physical examination revealed a hyperchromic patch with irregular borders and an erythematous halo, measuring about $1 \mathrm{~cm}$, at the site of the previous excision on the right lower quadrant of the abdomen (Figure 1). Dermoscopy revealed the predominance of a homogeneous dark brown pattern, with asymmetric and irregular borders (Figure 2). We performed an excisional biopsy and histopathological analysis revealed a junctional epidermal component with nests of mature nevus cells, as well as isolated nevus cells. We observed the presence

\footnotetext{
Received on 22.06.2016

Approved by the Advisory Board and accepted for publication on 12.09.2016

Work performed at Universidade Federal de Santa Maria (UFSM) - Santa Maria (RS), Brazil.

Financial support: none.

Conflict of interest: none.

1 Department of Clinical Medicine, Universidade Federal de Santa Maria (UFSM) - Santa Maria (RS), Brazil.

(C)2017 by Anais Brasileiros de Dermatologia
} 


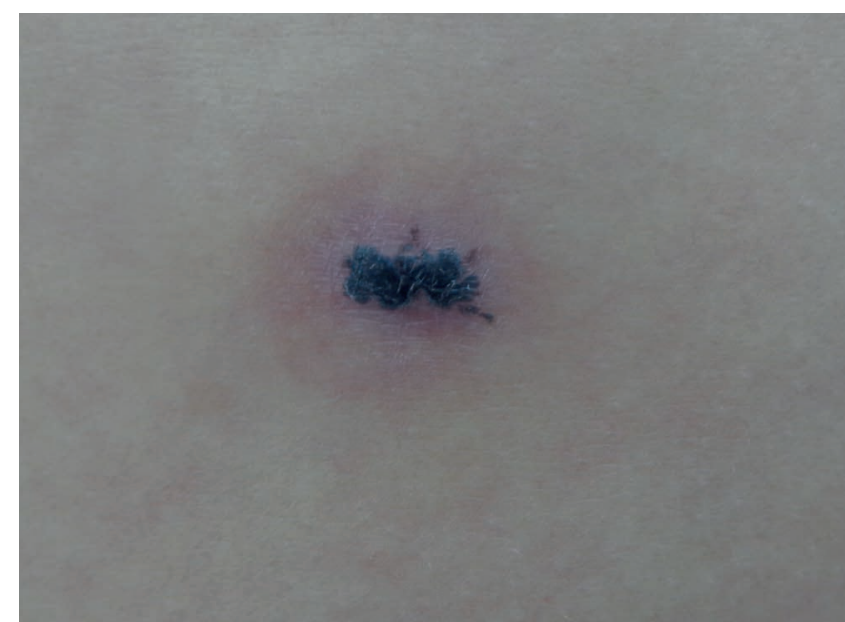

FIGURE 1: Right lower quadrant of the abdomen: irregular hyperchromic patch with erythematous halo, measuring about $1 \mathrm{~cm}$.

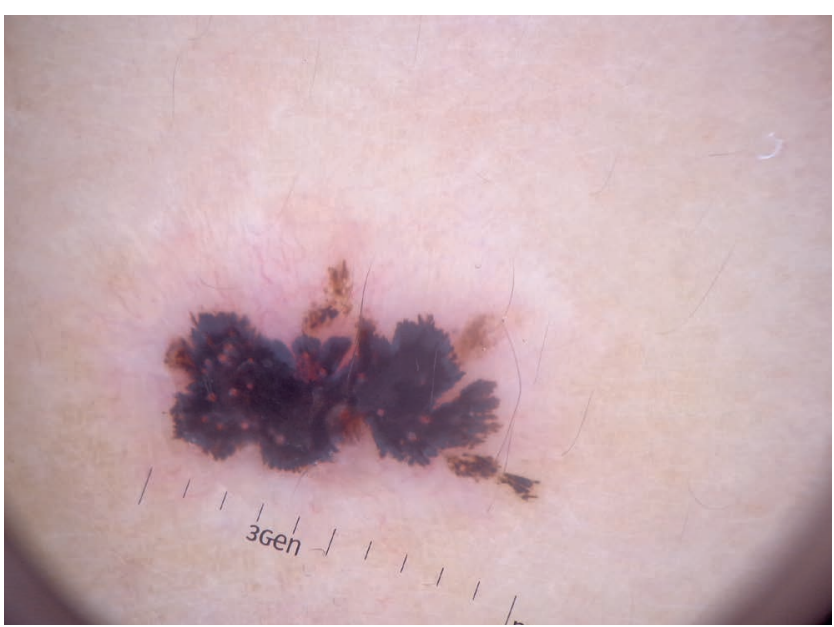

FIGURE 2: Dermoscopy: predominance of a blackish homogeneous pattern with asymmetric and irregular borders

of dermal fibrosis, melanophages with intracytoplasmic pigment and perivascular inflammatory infiltrate in the dermis, composed of lymphocytes and histiocytes. Epidermal changes did not extend beyond the area of dermal fibrosis-* (Figures 3, 4, and 5). Therefore, we diagnosed recurrent nevus.

\section{DISCUSSION}

Recurrent melanocytic nevi results from the proliferation of melanocytes remaining from a partially excised melanocytic nevi, leading to repigmentation at the site of previous excisions. It predominantly affects women aged 20-30 years, and the back is the most common site. On average, recurrence occurs six months after excision, and there is no consensus as to which type of nevus reccurs more often. ${ }^{1,3-6}$

Clinically, it manifests as a hyperpigmentation or hypopigmentation scarring area, linear striae, stippled halos, and/or diffuse pigmentation patterns. ${ }^{5}$ In many cases, melanocytic nevi recur

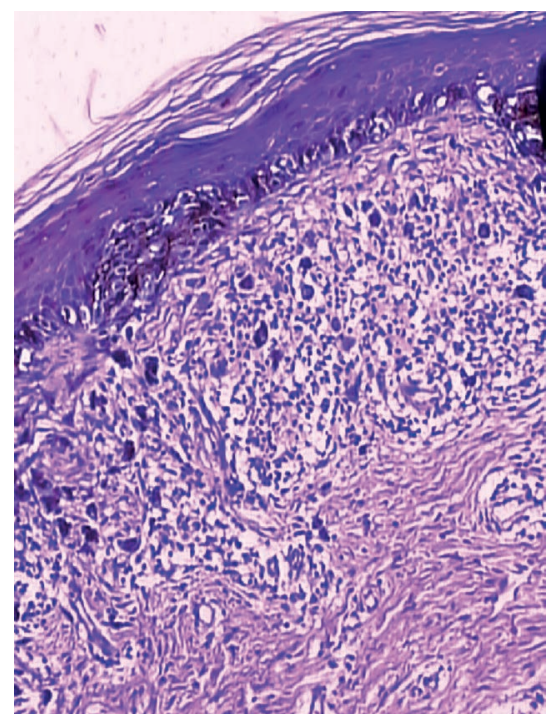

Figure 3: Histopathological examination of excisional biopsy (Hematoxylin \& eosin, X40)

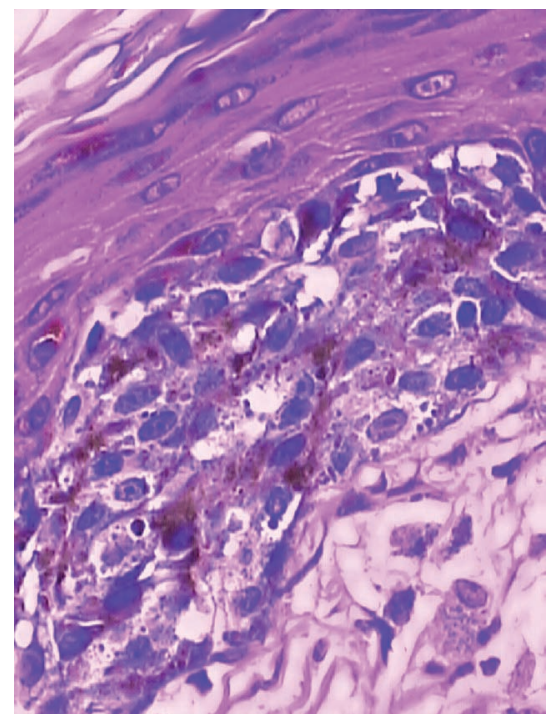

FIGURE 4: Dermal fibrosis, melanocytes with intracytoplasmic pigment and perivascular chronic inflammatory infiltrate (Hematoxylin \& eosin, X400)

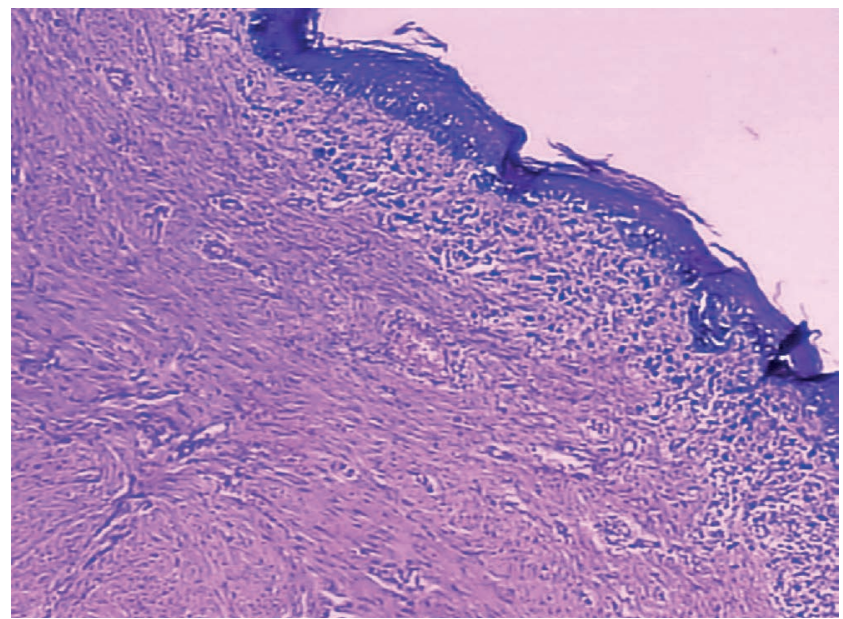

Figure 5: Junctional nests of mature nevus cells and some isolated nevus cells (Hematoxylin \& eosin, X40) 
asymmetrically with irregular pigmentation, which may lead to diagnostic confusion with melanoma. ${ }^{4}$

Histologically, melanocytic proliferation is observed at the dermoepidermal junction and in the superficial dermis, overlying the scarring area. In most cases, there is rectification of the epidermal ridges. Melanocytes are spindle cells, which may have mild to moderate cytologic atypia. However, mitotic activity is rarely observed. Contiguous involvement of the skin adjacent to the scar is often present. Differently, melanomas are characterized by greater degrees of cellular atypia, with ascending atypical melanocytes in the epidermis and cytological atypia in the dermal component. In addition, involvement of adnexal structures and lentiginous growth may occur. ${ }^{1}$ In the intermediate and late phases of melanoma regression, increased fibrosis, loss of melanocytic cells, and rectification of the epidermal crests occur. These characteristics may represent a challenge in the differential diagnosis between recurrent nevus and melanoma with regression. ${ }^{3}$

On dermoscopy, the presence of pigmentation beyond the border of the scar is the strongest indication for melanoma., ${ }^{2,6} \mathrm{In}$ addition, age over 30 years, location on the face and neck, chaotic pattern of growth, non-continuous growth in dermoscopy, and longer time interval between excision and recurrence are other fac- tors associated with malignancy. Dermoscopy - together with information such as the patient's age, anatomical location, recurrence time, growth pattern, and histological findings of the first excision - guides the final diagnostic interpretation. Unfortunately, it is often difficult or impossible to obtain the histopathological report of the initial lesion. In these cases of lesions with dubious dermoscopic findings, without previous history of excision or without previous histological report, the gold standard of treatment is the complete excision of the lesion. On the other hand, in cases with previous histological examination revealing melanocytic nevus, complete excision is indicated only for aesthetic reasons, and regular dermatoscopic follow-up is a possible and acceptable procedure. ${ }^{6}$

Recurrent melanocytic nevus usually occurs after incomplete removal inherent in procedures such as shave, punch, cryotherapy, or laser excisions. ${ }^{4}$ These procedures have good aesthetic results and can be used safely, without risk of malignancy. It should be emphasized that even when the initial diagnostic suspicion is of a benign lesion, it is important to send the excised material for histological examination and to inform the patient that incompletely removed lesions may reappear. This would avoid misdiagnosed suspicions of malignant melanoma and unnecessary physicians and patients' concerns. $\square$

\section{REFERENCES}

1. Brenn T. Pitfalls in the evaluation of melanocytic lesions. Histopathology. 2012;60:690-705

2. Longo C, Moscarella E, Pepe P, Cesinaro AM, Casari A, Manfredini M, et al. Confocal microscopy of recurrent naevi and recurrent melanomas: a retrospective morphological study. Br J Dermatol. 2011;165:61-8.

3. King R, Hayzen BA, Page RN, Googe PB, Zeagler D, Mihm MC Jr. Recurrent nevus phenomenon: a clinicopathologic study of 357 cases and histologic comparison with melanoma with regression. Mod Pathol. 2009;22:611-7

4. King R, Hayzen BA, Page RN, Googe PB, Zeagler D, Mihm MC Jr. Persistent melanocytic nevi: a review and analysis of 205 cases. Mod Pathol. 2009;22:611-7.

5. Fox JC, Reed JA, Shea CR. The Recurrent Nevus Phenomenon: A History of Challenge, Controversy, and Discovery. Arch Pathol Lab Med. 2011;135:842-6.

6. Blum A, Hofmann-Wellenhof R, Marghoob AA, Argenziano G, Cabo H, Carrera $C$, et al. Recurrent Melanocytic Nevi and Melanomas in Dermoscopy: Results of a Multicenter Study of the International Dermoscopy Society. JAMA Dermatol. 2014150:138-45.

\author{
MAILING ADDRESS: \\ Rafaella Daboit Castagna \\ Serviço de Dermatologia \\ Hospital Universitário de Santa Maria \\ Av. Roraima, 1.000, Prédio 22 \\ Camobi \\ 97105-900 Santa Maria, RS - Brazil \\ E-mail: lellacastagna@hotmail.com
}

How to cite this article: Castagna RD, Stramari JM, Chemello RML. The recurrent nevus phenomenon. An Bras Dermatol. 2017;92(4):531-3. 